> L'immunité humorale joue un rôle primordial dans la réponse contre les pathogènes, et le VIH (virus de l'immunodéficience humaine) ne déroge pas à cette règle. Si les anticorps dits neutralisants ont démontré leur efficacité de protection dans des modèles d'infection de macaques, leur induction est particulièrement difficile de par leurs caractéristiques très spécifiques. Parallèlement à la neutralisation, un panel d'activités inhibitrices complémentaires a été observé et suggère que des mécanismes d'inhibition dépendant de la région Fc des anticorps participent à la protection contre l'infection par le VIH, essentiellement au niveau des muqueuses. Ainsi, c'est cet ensemble d'activités neutralisantes et inhibitrices qui devra être considéré pour l'induction d'anticorps dans le cadre du développement de nouvelles stratégies vaccinales contre l'infection par le VIH. <

Fin 2010, le nombre de personnes vivant avec le VIH (virus de l'immunodéficience humaine) dans le monde était estimé à environ 34 millions (31,6-35,2 millions), soit une hausse de $17 \%$ par rapport à 2001 . Cette hausse reflète, d'une part, le nombre important et continu de nouvelles infections et, d'autre part, une extension de l'accès aux traitements antirétroviraux, qui a contribué à la réduction des décès liés au Sida (syndrome d'immunodéficience acquise). En 2009 et pour la première fois après 30 ans d'études sur le VIH, un essai vaccinal de phase III (RV144) a démontré une efficacité de protection de $31 \%$ contre l'infection [1]. Si ce pourcentage reste faible, il s'agit cependant du premier essai vaccinal démontrant une protection contre le VIH.

Il est désormais admis que l'induction d'anticorps neutralisants ( $\mathrm{AcN}$, voir Glossaire) spécifiques et la stimulation de l'ensemble des mécanismes dépendant des anticorps sont des prérequis au développement d'un

\section{Les anticorps anti-VIH}

\section{De multiples activités antivirales}

Maryse Peressin ${ }^{1}$, Vincent Holl ${ }^{2}$, Christiane Moog ${ }^{1}$

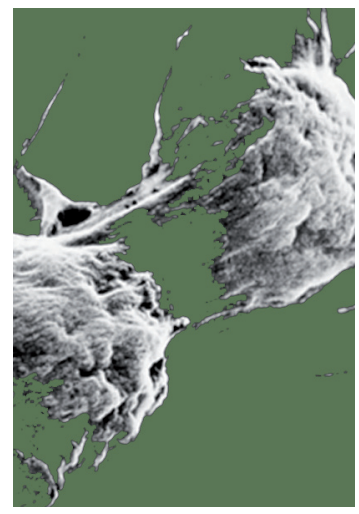

${ }^{1}$ Inserm U1110,

Université de Strasbourg,

3 , rue Koeberlé, 67000 Strasbourg, France ; ${ }^{2}$ COVANCE, Lab Science department, hematology and flow department, 7 , rue Moise-Marcinhes, 1217 Meyrin, Genève, Suisse. c.moog@unistra.fr

vaccin contre le VIH. Les dernières générations de vaccin à l'étude sont basées sur l'utilisation d'immunogènes multiples en prime-boost (voir Glossaire) capables d'activer et d'amplifier en parallèle les deux bras de la réponse immunitaire spécifique - humorale et cellulaire. Ce type de stratégie a été expérimenté dans l'essai thaïlandais RV144 (immunisation avec la combinaison de vecteurs viraux ALVAC ${ }^{\text {TM }}$ [produit par Sanofi-Pasteur] et de protéine gp120 [voir Glossaire] recombinante AIDSVAX ${ }^{\circledR} B / \varepsilon$ [développé par VaxGen]). Pourtant, dans cet essai, ni les anticorps ayant une activité neutralisante, ni la réponse cellulaire, n'ont pu être corrélés avec la protection. Cette dernière a, au contraire, été associée à l'induction d'anticorps non neutralisants inhibiteurs (AcNoNI, voir Glossaire) dirigés contre la boucle V1/V2 de la gp120 [1]. Ces anticorps particuliers ont démontré des activités inhibitrices dépendantes de la reconnaissance d'épitopes sur une cellule infectée d'une part, et d'autre part de la fixation de leur région Fc sur les récepteurs Fc (RFc, voir Glossaire) exprimés par des cellules effectrices [2]. Si la découverte d'immunogènes capables d'induire in vivo des anticorps de type neutralisants constituerait une avancée majeure dans le domaine de la recherche vaccinale, les résultats de l'essai RV144 ont démontré que l'induction d'anticorps de types inhibiteurs était non seulement possible, mais également corrélée avec la protection contre l'infection [1, 3, 4]. Ainsi, des activités inhibitrices distinctes de la neutralisation classique joueraient un rôle important dans le contrôle de l'infection. La mesure de ce type d'activité était jusqu'alors largement sous-évaluée dans le contexte de la réponse antivirale en général, et de la réponse anti-VIH en particulier.

Dans cette revue, le point sera fait sur l'état des connaissances concernant le rôle et les mécanismes de neutralisation et d'inhibition liés aux anticorps dans la protection contre le VIH in vitro et in vivo, ainsi que dans l'élaboration de stratégies vaccinales innovantes. 


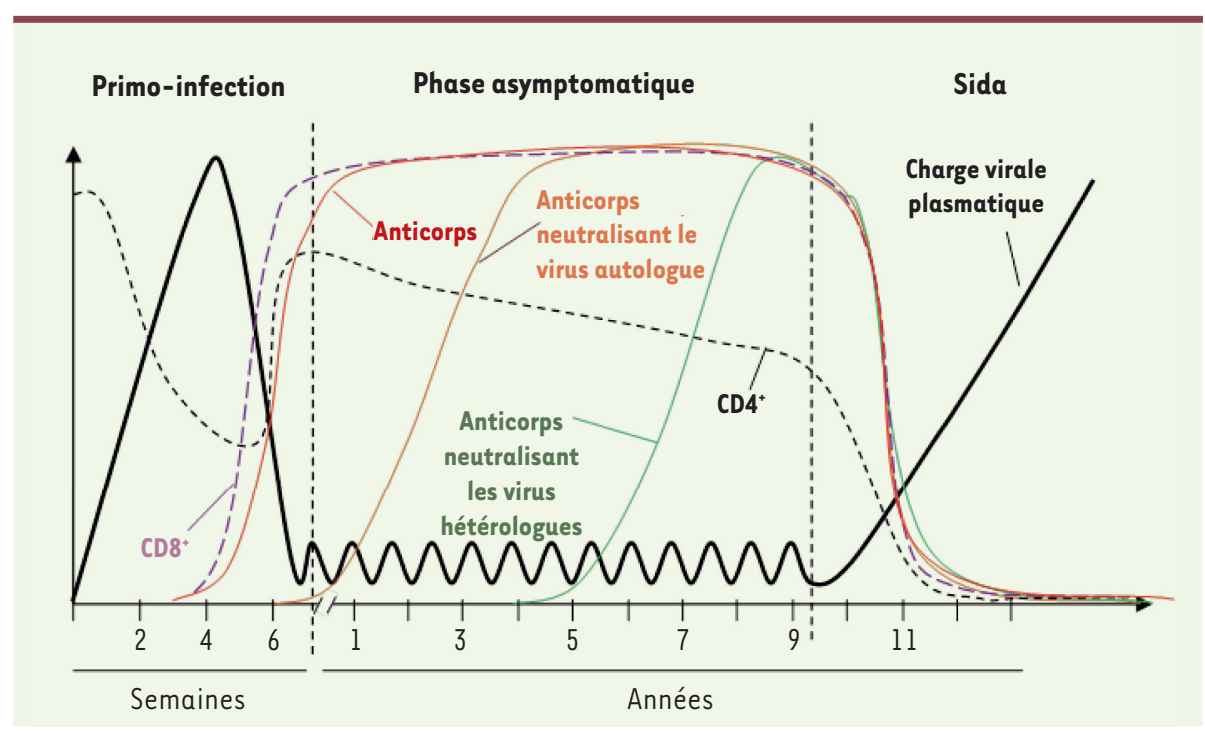

Figure 1. Évolution de l'infection par le VIH-1. Représentation schématique des trois phases de l'infection par le VIH-1. Les différentes courbes symbolisent l'évolution de différents facteurs viraux, cellulaires et humoraux en fonction du temps suivant l'infection.

la gp4l (Figure 2). Plus récemment, et grâce à de nouvelles techniques de criblage, d'autres Ac neutralisants dirigés contre la gp120 ont pu être détectés $[7,8]$.

Ces Ac neutralisants ont des caractéristiques très particu-

\section{Infection par le VIH et réponse humorale}

Après une infection par le VIH-1, on distingue trois phases dans l'évolution de la maladie, présentant des caractéristiques virologiques et immunologiques distinctes (Figure 1). La primo-infection se caractérise par un pic de la charge virale plasmatique et des perturbations importantes du nombre et de la composition des cellules mononucléées du sang périphérique. La chute de la virémie plasmatique est consécutive à l'apparition de la réponse cytotoxique (cellules natural killer [NK] et lymphocytes T CD8) et des anticorps $(A C)$ spécifiques du virus (séroconversion concomitante du début de la phase asymptomatique). Les anticorps produits sont de type immunoglobulines (Ig) G et IgA ; ils reconnaissent l'ensemble des protéines virales. Cependant, si la réponse humorale spécifique est en principe forte et persistante, avec le développement d'une réponse humorale autologue dès la première année après la séroconversion [5] (courbe orange, Figure 1), seuls 10 à $20 \%$ des patients séropositifs développent des anticorps hétérologues possédant une activité neutralisante vis-à-vis d'un large spectre de souches virales (courbe verte, Figure 1). Ces Ac neutralisants sont le résultat de la maturation de la réponse immune du fait de contacts répétés avec de nombreux épitopes de la gp120 après plusieurs années d'infection. L'apparition de ce type d'anticorps n'est pas nécessairement associée à une évolution favorable de la maladie. En effet, une fois l'infection établie, la capacité de contrôle de la réplication du VIH par les anticorps neutralisants est faible, en raison des échappements perpétuels du virus et de l'établissement de réservoirs viraux $[6,40]$.

\section{Mécanisme de neutralisation, activité neutralisante}

Initialement, cinq anticorps monoclonaux capables de neutraliser un large spectre d'isolats primaires du VIH (souches isolées de patients infectés) ont été identifiés (b12, 447-52D, 2G12, 4E10 et $2 F 5$ ). Ils reconnaissent des épitopes spécifiques de la gp 120 ou de lières et sont généralement le résultat d'une longue maturation, traduit par un nombre élevé de mutations somatiques (Figure 2). De plus, certains possèdent une longue chaîne lourde HCDR3, capable de reconnaître des épitopes enfouis, peu accessibles mais conservés [9]. Cette grande complexité, ainsi que leur conformation tout à fait particulière, rendent leur induction par vaccination extrêmement complexe.

Le rôle des Ac neutralisants a été largement étudié in vivo dans des modèles animaux, principalement dans des modèles de primates non humains (NHP) [10, 11]. L'utilisation de primates non humains infectés de manière expérimentale par voie intraveineuse, orale ou par les muqueuses, avec des virus SHIV (voir Encadré 1) a permis de démontrer le rôle protecteur de ces anticorps. Le transfert passif d'Ac neutralisants (inoculés par voie intraveineuse) a permis de protéger de l'infection, c'est-à-dire conférer une immunité stérilisante [10, 11]. L'ensemble des résultats obtenus à l'aide des modèles de primates non humains indique que l'induction d'Ac neutralisants par vaccination permettrait de protéger de l'infection par le VIH.

\section{Activités inhibitrices des anticorps}

Des anticorps dépourvus d'activité neutralisante, mais exerçant une activité inhibitrice, ont été identifiés [12]. L'activité anti-VIH de ces anticorps, dénommés anticorps non neutralisants inhibiteurs ( $A c N o N I)$, est strictement dépendante de la liaison entre leur région Fc et un récepteur Fc exprimé à la surface d'une cellule effectrice. Différents mécanismes d'inhibition ont été démontrés in vitro (Figure $3 B-C$ ). 
A

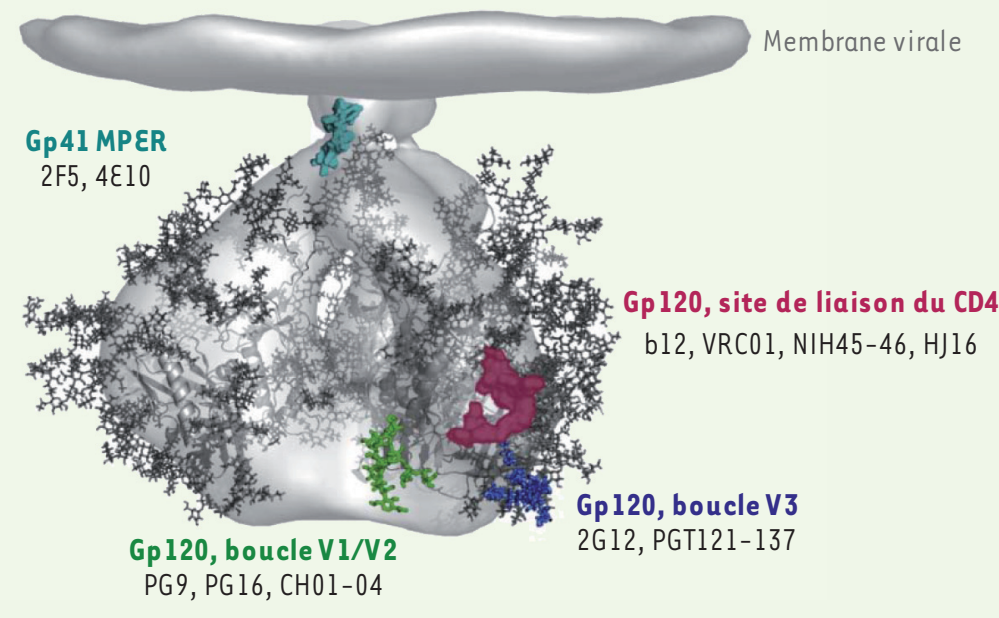

B

\begin{tabular}{|c|c|c|c|}
\hline $\begin{array}{l}\text { Anticorps } \\
\text { neutralisant }\end{array}$ & Épitope reconnu & $\begin{array}{l}\text { Longueur chaîne } \\
\text { lourde CDR3 (aa*) }\end{array}$ & $\begin{array}{c}\text { Pourcentage } \\
\text { de mutation } V_{H}(\%) *\end{array}$ \\
\hline $2 F 5$ & gp4l, MPER & 24 & 15.2 \\
\hline $4 \varepsilon 10$ & gp4l, MPER & 20 & 15.6 \\
\hline $2 \mathrm{G} 12$ & gp120, mannoses conservés boucle V3 & 16 & 31.7 \\
\hline PGT128 & gp120, glycane boucle V3 & 19 & 19 \\
\hline $\operatorname{lgGl~bl2~}$ & gp120, site de liaison au CD4 & 20 & 13.1 \\
\hline VRCOl & gpl20, site de liaison au CD4 & 14 & 32.1 \\
\hline $\mathrm{NIH} 45-46$ & gp120, site de liaison au CD4 & 18 & 35.7 \\
\hline HJl6 & gp120, site de liaison au CD4 & 21 & 14.6 \\
\hline PG9/PGl6 & gp120, boucle V1/V2 gpl20 & 30 & $16.7-20.5$ \\
\hline $\mathrm{CHOl-04}$ & gp120, boucle V1/V2 gp120 & 24 & $11.5-14.3$ \\
\hline IgG donneur sain & Non spécifique du VIH & 15 & 5.9 \\
\hline
\end{tabular}

Figure 2. Complexité des anticorps neutralisants. A. Représentation schématique du trimère de glycoprotéines d'enveloppe du VIH-1; en couleur les sites de reconnaissance des principaux anticorps neutralisants. B. Caractéristiques fonctionnelles des principaux anticorps neutralisants; aa : acides aminés ; * : estimation basée sur la comparaison de séquences $V(D) J$ entre l'anticorps neutralisant (muté) et son anticorps «origine 》 (non muté) (adaptée de [32]).

\section{Activité inhibitrice dépendante de la fixation de la région Fc des IgG aux RFc $\gamma$ des cellules présentatrices d'antigènes}

Un mécanisme d'inhibition du VIH par les anticorps impliquant l'interaction du fragment constant ( $F c$ ) d'une IgG à un RFc $\gamma$ a été mis en évidence in vitro $[12,13]$ (Figure 3). En effet, lorsque des cellules présentatrices d'antigène (cellules dendritiques [DC] ou macrophages), qui expriment les récepteurs au Fc de type $\gamma(\mathrm{RF} \gamma \gamma)$, sont les cibles du VIH plutôt que des lymphocytes T CD4 (dépourvus de RFc $\gamma$ ), l'activité inhibitrice des IgG est augmentée de façon drastique (100 à 1000 fois pour les DC ou les macrophages, respectivement $[13,14])$. Le mécanisme d'inhibition n'a pas encore été clairement identifié même s'il a été proposé que les complexes immuns, fixés aux cellules effectrices par l'intermédiaire des RFc $\gamma$, seraient phagocytés puis dégradés dans des lysosomes spécifiques. La fixation des anticorps sur les RFc de cellules effectrices peut aussi déclencher l'activation d'autres mécanismes fonctionnels qui pourraient conduire à une diminution de la réplication virale, comme:

- l'induction de cytokines et/ou de chimiokines antivirales [15];

- la maturation des cellules présentatrices d'antigènes qui favoriserait la restriction virale par des mécanismes postfusion [16]. 
L'ADCC ou la réponse cytotoxique cellulaire dépendante des anticorps

La cytotoxicité à médiation cellulaire dépendante des anticorps $(A D C C)$ est un mécanisme combinant des composants de l'immunité innée et adaptative : les anticorps qui reconnaissent la cellule infectée se fixent, via le RFc, à une cellule effectrice (généralement une cellule NK) impliquée dans la défense innée (Figure 3C). Cette interaction entre la cellule infectée et la cellule effectrice par l'intermédiaire de l'anticorps entraîne l'activation de la cellule effectrice qui libère des granules cytotoxiques (granzyme et perforine), des chimiokines, de l'oxyde nitrique ou des dérivés réactifs de l'oxygène conduisant à la destruction de la cellule infectée (Figure 3C) [17].

\section{Les anticorps non neutralisants inhibiteurs in vivo}

Les Ac non neutralisants inhibiteurs ont suscité un regain d'intérêt suite aux résultats de l'essai clinique RV144, où un corrélat de protection a été mis en évidence avec l'induction de ce type d'anticorps inhibiteurs. Il est cependant à noter que l'importance des mécanismes d'inhibition dépendants des RFc a d'ores et déjà été confirmé dans des modèles animaux $[11,18,19]$. En effet, dans un modèle de primate non humain, l'étude du transfert passif de l'anticorps neutralisant b12 ou de l'anticorps b12 mutant (dénommé LALA et incapable de fixer le complément ou les $R F c \gamma$ ) a démontré une diminution de $50 \%$ de l'efficacité de protection par l'anticorps LALA [11, 18]. Plus récemment, une fonction de protection partielle et une activité sur la diminution de la charge virale ont été attribuées à des Ac non neutralisants inhibiteurs dans le modèle de primate non humain. Ces anticorps possèdent des fonctions inhibitrices dépendantes de leur région $\mathrm{Fc}$ et de l'expression des RFc [19, 20].

Chez les patients infectés, des anticorps inhibiteurs sont fréquemment détectés très tôt après la phase aiguë de l'infection (Figure 1 , courbe rouge) $[21,22]$. De manière intéressante, une activité inhibitrice dépendante des RFc est détectée de manière significativement plus élevée dans une cohorte de patient dits «contrôleurs 》 ou «élites » (patients ayant une virémie indétectable pendant plusieurs années en absence de tout traitement) par rapport aux patients virémiques [23]. Des études supplémentaires seront nécessaires afin de déterminer si les activités inhibitrices détectées chez les patients infectés sont la cause ou la conséquence du contrôle de l'infection et de l'évolution plus lente vers la maladie.

L'ensemble des résultats suggèrent qu'un vaccin devrait induire des anticorps inhibiteurs impliquant les récepteurs $\mathrm{Fc}$ en plus des anticorps neutralisants, afin de solliciter au maximum l'ensemble des mécanismes de défense contre l'infection. Les anticorps inhibiteurs auraient pour avantage de reconnaître des sites hautement immunogènes, et pourraient de ce fait être plus facilement inductible par vaccination.

\section{Le cas particulier des IgA}

La réponse IgA spécifique se développe parallèlement à la réponse IgG chez les patients infectés par le VIH-l. Cependant, les réponses relayées par les IgA spécifiques du virus sont faibles, tant au niveau des muqueuses qu'au niveau systémique [24]. De façon intéressante, des observations réalisées chez des sujets séronégatifs partenaires de sujets séropositifs HEPS (highly exposed persistently seronegative) suggèrent que des IgA reconnaissant le VIH sont détectées dans le tractus génital ou le liquide séminal, et cela en l'absence de sérologie IgG anti-VIH positive $[25,41]$. Par ailleurs, des IgA spécifiques du virus ont été détectées dans les sécrétions salivaires de certains nourrissons dont les mères étaient infectées par le VIH. La présence de ces IgA chez des sujets séronégatifs en contact régulier avec le VIH questionne sur leur rôle potentiel dans la protection.

In vitro, il a été observé que pour un épitope donné (celui reconnu par l'Ac neutralisant b12), l'activité neutralisante d'une IgA était équivalente à celle d'une IgG [26]. Cependant, les fonctions cellulaires impliquant la région Fc des IgA sont différentes de celles impliquant la région Fc des IgG. Dès lors, la présence d'IgA au niveau des muqueuses engagerait l'activation locale de mécanismes immunitaires distincts de ceux qu'induisent les IgG, tels que l'agrégation des IgA sécrétoires [27] ou des mécanismes de type ADCC, impliquant des cellules effectrices (telles que des polynucléaires neutrophiles) exprimant des RFc $\alpha$.

Si pour l'instant le nombre d'études analysant les IgA reste limité, les résultats suggèrent qu'elles pourraient porter des fonctions spécifiques qui s'ajouteraient à celles des IgG et, ainsi, participer à la protection contre I'infection par le VIH au niveau des muqueuses [28].

\section{Effet délétère des anticorps anti-VIH : les anticorps facilitants}

Différent travaux réalisés sur des lignées cellulaires ont mis en évidence des anticorps nommés «facilitants ». Ces anticorps, contrairement aux anticorps neutralisants ou inhibiteurs, faciliteraient l'infection par le VIH. Le mécanisme de facilitation serait lié in vitro à une augmentation soit du titre viral (augmentation du nombre de cellules infectées), soit de la production de particules virales infectieuses. Le mécanisme responsable de cette augmentation n'est pas identifié à I'heure actuelle, mais il impliquerait les RFc [29].

In vivo, une corrélation entre la présence d'anticorps facilitants et une augmentation de la sévérité de la maladie a été observée pour le virus de la dengue [30]. Néanmoins, aucun rôle facilitateur des anticorps n'a pu être mis en évidence in vivo pour d'autres infections virales en général, ni pour le VIH en particulier [4]. Cependant, il est à noter qu'une étude récente a 


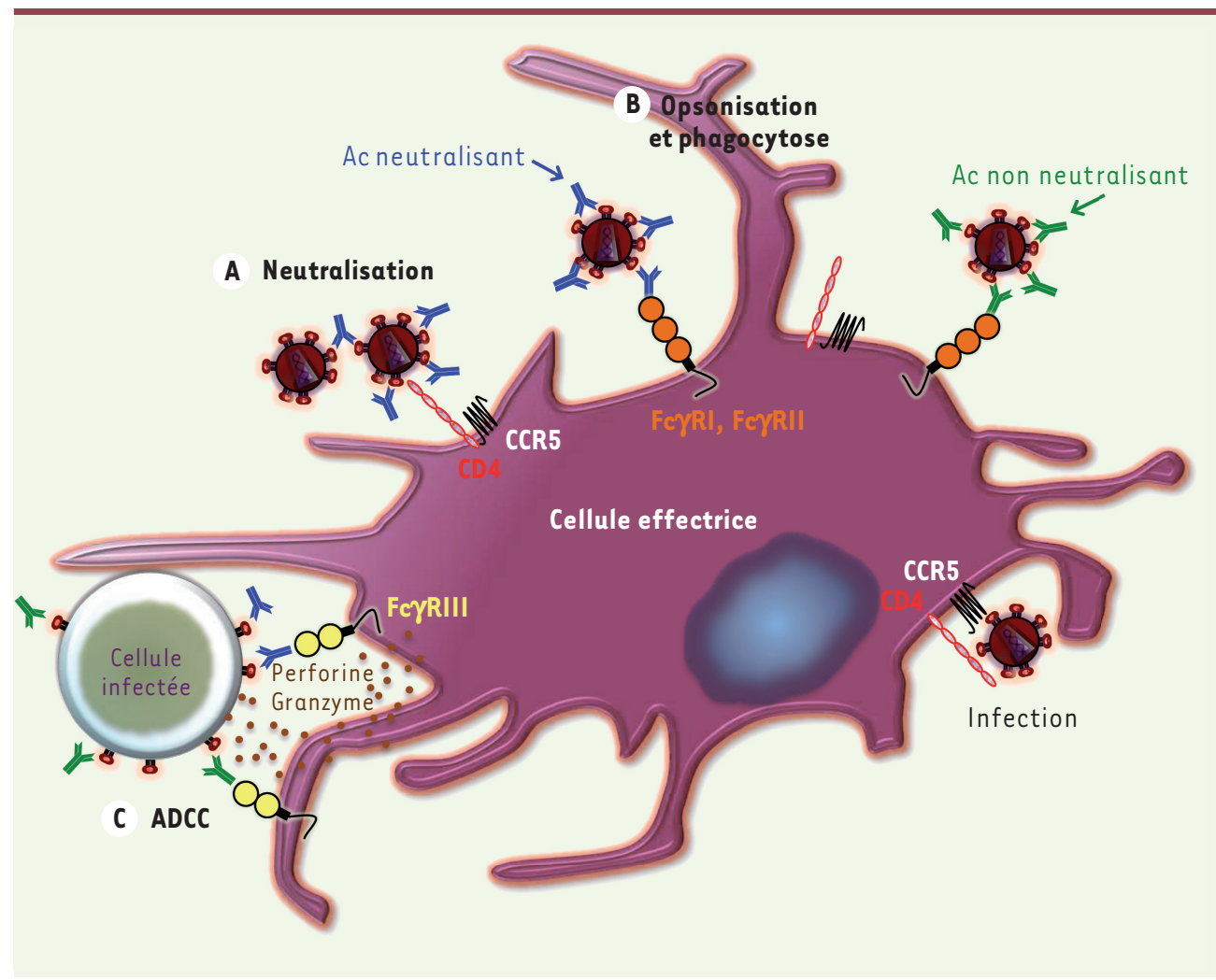

Figure 3. Différents mécanismes d'inhibition du VIH-1 par les anticorps. A. Neutralisation des particules virales par les anticorps neutralisants. B. Opsonisation des particules virales par les anticorps (neutralisants ou non neutralisants). La liaison de la région Fc de l'anticorps avec le RFc d'une cellule effectrice induit la phagocytose des complexes immuns. C. L'ADCC (cytotoxicité à médiation cellulaire dépendante des anticorps) correspond à la reconnaissance d'une cellule infectée par un anticorps. La fixation au RFc spécifique sur une cellule effectrice conduit à la lyse de la cellule infectée.

démontré une corrélation entre la présence d'un allèle particulier du $R F c \gamma$ et une augmentation du risque d'infection dans un sous-groupe de volontaires ayant de faibles pratiques à risques [31]. Ces résultats suggèrent un possible effet délétère d'anticorps spécifiques du VIH chez une sous-population de patients ayant un génotype particulier de RFc $\gamma$. Des études complémentaires seront nécessaires afin de confirmer cette association, et il s'agira de ne pas sous-estimer ce type d'effets dans le futur développement de vaccins prophylactiques.

\section{Vers de nouvelles pistes vaccinales}

Dans le développement d'un vaccin contre le VIH, les espoirs fondés sur l'induction d'anticorps de type neutralisants ont été compromis par la découverte de leurs caractéristiques particulières (Iongue chaîne lourde HCDR3, nombre important de mutations somatiques) nécessitant une longue maturation et rendant ainsi leur induction très difficile (Figure 2). Cette maturation des lymphocytes B ne pourra vraisemblablement pas être reproduite par une stimulation courte avec un immunogène unique [32]. Ainsi, des stratégies vaccinales fondées sur une succession d'immunogènes capables de mimer, étape par étape, les phénomènes de maturation et d'activation des clones de cellules
B sont aujourd'hui proposées. Ce type de vaccination commence tout juste à être expérimenté mais, compte tenu de la complexité des mécanismes impliqués, les chances de succès sont loin d'être acquises.

Au vu des contraintes importantes liées à l'induction d'anticorps neutralisants in vivo, les recherches vaccinales s'orientent vers l'optimisation des caractéristiques des anticorps et l'induction de mécanismes immunitaires additionnels, ainsi que vers des méthodes permettant l'amélioration de la réponse immune. $\varepsilon$ n suivant des concepts développés dans le domaine de la lutte antitumorale, de nombreuses études se sont focalisées sur la modification de la région $\mathrm{Fc}$ des anticorps afin d'en accroître l'activité inhibitrice. L'équipe de Burton a généré par mutagenèse dirigée ou par modification de la glycosylation de la région $\mathrm{Fc}_{c}$ [42], un panel d'Ac mutants de l'Ac neutralisant $b 12$ possèdant des affinités variables pour les RFc $\gamma$, tout en conservant l'activité neutralisante de la région Fab b12 [33, 34]. Ces mutations ont permis d'augmenter l'affinité des anticorps pour les RFc $\gamma$ et, de ce fait, les fonctions inhibitrices associées (phagocytose, $A D C C$ ) in vitro, sans améliorer la protection in vivo dans des expériences d'infections expérimentales chez le macaque. Ce type d'anticorps devrait être induit directement au niveau des sites d'infections (muqueuses anales, vaginales, etc.) afin de limiter la réplication virale dans les nombreuses cellules cibles présentes. C'est pourquoi de nouveaux immunogènes sont actuellement formulés afin d'induire une réponse humorale directement au niveau de ces muqueuses [35]. Un essai clinique de phase I a récemment débuté (projet collaboratif européen EuroNeut41) dans le but de tester ces nouveaux concepts [36]. 


\section{Modèle de primate non humain pour l'étude des anticorps protecteurs anti-VIH}

Les macaques (Rhésus ou Cynomolgus) infectés par un virus simien (SIV) développent un Sida proche de celui qu'induit le VIH chez I'homme. Afin de pouvoir analyser le rôle de la réponse humorale face à l'infection par le VIH dans ce modèle animal, différents virus chimériques (SHIV) ont été construits. Pour ces virus, l'enveloppe du SIV a été remplacée par celle d'un VIH-l. Les virus SHIV reproduisent chez le singe la physiopathogénie du VIH chez l'homme. La présence de l'enveloppe du VIH permet l'induction et l'étude d'une réponse humorale spécifique dirigée contre l'enveloppe du virus humain. Les virus chimériques constituent donc un modèle de choix pour l'étude du rôle des anticorps anti-VIH dans la protection contre l'infection.

Différents modèles d'infection expérimentale SHIV ont été développés (dose unique forte ou succession de doses faibles répétées, infection par voies vaginale, rectale ou systémique), permettant ainsi l'étude du rôle des anticorps et des corrélats de protection.

Ainsi, les modèles de primate non humain ont largement contribué à la compréhension des mécanismes d'inhibition du virus par les anticorps et constituent des prérequis pour nous guider dans le choix et le développement d'un vaccin prophylactique contre le VIH.

Nos connaissances actuelles des mécanismes d'action des anticorps anti-VIH restent cependant très incomplètes, et des résultats récents leur attribuent des activités complémentaires plus larges. Le groupe de Haigwoog souligne une augmentation d'une réponse lymphocytaire $B$ spécifique, en réponse au transfert passif d'anticorps anti-VIH dans un modèle de primate non humain [37]. Dans un modèle de rétrovirus murin FrCas, Michaud et al. observent une protection liée à l'induction d'une réponse $B$ et $T$ à long terme, après le transfert passif d'anticorps neutralisants [38]. Ce type de mécanisme de stimulation de la réponse adaptative par des anticorps a également été proposé précédemment dans le domaine du cancer $[39,43]$. Ces études attribuent aux anticorps une fonction supplémentaire «d'immunogène » qui induirait une réponse primaire, puis mémoire, plus efficace qu'une particule virale libre ou qu'une cellule infectée. Les Ac participeraient ainsi à la mise en place d'une réponse adaptative, ouvrant la voie vers de nouveaux champs d'application.

\section{Conclusion}

Près de 30 ans après la découverte du VIH, de nombreux concepts vaccinaux ont été testés et les premiers résultats de protection partielle viennent tout juste d'être démontrés. Le rôle des anticorps dans la protection contre le VIH est indéniable, et cette protection est composée d'un ensemble complexe de mécanismes faisant intervenir différents types d'anticorps, de récepteurs, de cellules effectrices, mais aussi le déclenchement d'autres composants du système immunitaire inné, adaptatif et mémoire. Nos connaissances acquises dans les domaines de l'infection et de la réponse immune antivirale nous permettent à présent de proposer de nouvelles voies à explorer dans

\section{GLOSSAIRE}

Anticorps neutralisant (AcN) : anticorps ayant la capacité d'inhiber in vitro la réplication virale dans une cellule cible et ceci en l'absence d'autres composants comme les récepteurs $\mathrm{Fc}$, le complément ou des cellules effectrices. La reconnaissance d'une particule virale par la région Fab d'un anticorps neutralisant inhibe la réplication du virus en empêchant des évènements précoces comme la fixation ou la fusion virus/cellule hôte.

Anticorps non neutralisant inhibiteur (AcNoNI) : anticorps dépourvu d'activité neutralisante, mais ayant la capacité d'inhiber la réplication virale par l'intermédiaire d'autres composants, tels que les récepteurs $\mathrm{Fc}$, le complément ou des cellules effectrices. Un tel anticorps reconnaît la particule virale (ou une cellule infectée) via sa région Fab, mais l'activité inhibitrice est dépendante de mécanismes liés à sa région Fc.

Gp120 : glycoprotéine de surface de 120 kDa exprimée par le VIH, constituée de cinq épitopes variables (VI, V2, V3, V4 et V5). Les boucles V1 et V2, très flexibles, masquent le site de fixation au récepteur CD4. De nombreux anticorps anti-VIH sont dirigés contre la gpl20. Cependant, l'hypervariabilité de cette protéine permet d'échapper à la réponse humorale spécifique.

Récepteurs $\mathrm{Fc}(\mathrm{RFc})$ : récepteurs exprimés à la surface des diverses cellules du système immunitaire (neutrophiles, macrophages, cellules dendritiques) capables de fixer la région Fc des anticorps. Les RFc se distinguent par leur affinité pour les différents types d'immunoglobulines (RFc $\gamma$ I, II ou III pour les IgG, RFc $\alpha$ pour les IgA, etc.) et leurs différentes sous-classes (IgGl, IgG2, etc.). La fixation d'un anticorps à un RFc déclenche une cascade de signaux intracellulaires conduisant à l'activation de la cellule effectrice.

Vaccination prime-boost (littéralement amorce-rappel) : stratégie de vaccination qui permet de présenter des sites antigéniques sous des formes différentes par différents vecteurs et de façon séquentielle. Ce mode de vaccination permet de stimuler de manière successive les différents bras de la réponse immune (humorale et cellulaire).

l'espoir de développer une réponse antivirale forte et complète, nécessaire au développement d'un vaccin prophylactique efficace contre le VIH. $\diamond$

\section{SUMMARY}

\section{Anti-HIV antibodies: multiple antiviral activities}

Sexual transmission is currently the major route of HIV infection worldwide. Neutralizing antibodies ( $\lg G$ ) have demonstrated their role in the protection from experimental challenge in non-human primate's model. However, these types of antibodies display very specific characteristics and are extremely difficult to 
induce. Interestingly, antibodies devoid of neutralizing activity have demonstrated additional inhibitory mechanisms dependant of their binding to Fc receptors expressed on antigen presenting cells. These cells may play decisive role at early sexual transmission as they have been proposed to be the first HIV target at the mucosal site. Data from in vivo studies and recent findings following clinical assays demonstrated the importance of these Fc-mediated antibodies dependant mechanism in protection against HIV. Therefore new vaccination strategies including the induction of such type of activities, in addition to neutralizing antibodies, should be developed. $\diamond$

\section{LIENS D'INTÉRÊT}

Les auteurs déclarent n'avoir aucun lien d'intérêt concernant les données publiées dans cet article.

\section{RÉFÉRENCES}

1. Haynes BF, Gilbert PB, McElrath MJ, et al. Immune-correlates analysis of an HIV-1 vaccine efficacy trial. N Engl J Med $2012 ; 366: 1275-86$.

2. Tomaras GD, Ferrari G, Shen X, et al. Vaccine-induced plasma IgA specific for the $\mathrm{Cl}$ region of the HIV-1 envelope blocks binding and effector function of IgG. Proc Natl Acad Sci USA 2013; 110 : 9019-24.

3. McMichael AJ, Haynes BF. Lessons learned from HIV-1 vaccine trials: new priorities and directions. Nat Immunol $2012 ; 13: 423-7$.

4. Montefiori DC, Karnasuta C, Huang $Y$, et al. Magnitude and breadth of the neutralizing antibody response in the RV144 and Vax003 HIV-1 vaccine efficacy trials. J Infect Dis 2012 ; 206 : 431-41.

5. Mascola JR, Montefiori DC. The role of antibodies in HIV vaccines. Annu Rev Immunol $2010 ; 28$ : 413-44.

6. Moog C, Fleury HJ, Pellegrin I, et al. Autologous and heterologous neutralizing antibody responses following initial seroconversion in human immunodeficiency virus type l-infected individuals. J Virol $1997 ; 71: 3734-41$

7. Walker LM, Huber M, Doores KJ, et al. Broad neutralization coverage of HIV by multiple highly potent antibodies. Nature $2011 ; 477: 466-70$.

8. Scheid JF, Mouquet $\mathrm{H}$, Feldhahn N, et al. Broad diversity of neutralizing antibodies isolated from memory B cells in HIV-infected individuals. Nature $2009 ; 458: 636-40$.

9. Kwong PD, Mascola JR. Human antibodies that neutralize HIV-l: identification, structures, and B cell ontogenies. Immunity $2012 ; 37: 412-25$.

10. Mascola JR, Stiegler G, VanCott TC, et al. Protection of macaques against vaginal transmission of a pathogenic HIV-1/SIV chimeric virus by passive infusion of neutralizing antibodies. Nat Med $2000 ; 6: 207-10$.

11. Hessell AJ, Poignard P, Hunter M, et al. Effective, low-titer antibody protection against low-dose repeated mucosal SHIV challenge in macaques. Nat Med 2009 ; 15 : 951-4.

12. Holl V, Peressin M, Decoville $T$, et al. Nonneutralizing antibodies are able to inhibit human immunodeficiency virus type 1 replication in macrophages and immature dendritic cells. J Virol $2006 ; 80: 6177-81$.

13. Holl V, Peressin M, Schmidt S, et al. Efficient inhibition of HIV-1 replication in human immature monocyte-derived dendritic cells by purified anti-HIV-1 IgG without induction of maturation. Blood $2006 ; 107:$ 4466-74.

14. Peressin M, Holl V, Schmidt S, et al. HIV-1 replication in Langerhans and interstitial dendritic cells is inhibited by neutralizing and Fc-mediated inhibitory antibodies. J Virol $2011 ; 85: 1077-85$.

15. Moody MA, Liao HX, Alam SM, et al. Anti-phospholipid human monoclonal antibodies inhibit CCR5-tropic HIV-1 and induce beta-chemokines. J Exp Med 2010 ; 207 : 763-76.

16. Su B, Xu K, Lederle A, et al. Neutralizing antibodies inhibit HIV-l transfer from primary dendritic cells to autologous CD4 T-lymphocytes. Blood $2012 ; 120: 3708-17$.

17. Huber M, Trkola A. Humoral immunity to HIV-1: neutralization and beyond. J Intern Med 2007 ; $262: 5-25$.

18. Hessell AJ, Hangartner L, Hunter $M$, et al. Fc receptor but not complement binding is important in antibody protection against HIV. Nature 2007 ; 449 : 101-4.

19. Burton DR, Hessell AJ, Keele BF, et al. Limited or no protection by weakly or nonneutralizing antibodies against vaginal SHIV challenge of macaques compared with a strongly neutralizing antibody. Proc Natl Acad Sci USA 2011 ; 108 : 11181-6.

20. Moog C, Dereuddre-Bosquet N, Teillaud JL, et al. Protective effect of vaginal application of neutralizing and nonneutralizing inhibitory antibodies against vaginal SHIV challenge in macaques. Mucosal Immunol 2013 ; doi : 10.1038/mi.2013.23.

21. Connick $\varepsilon$, Marr DG, Zhang XQ, et al. HIV-specific cellular and humoral immune responses in primary HIV infection. AIDS Res Hum Retrovir 1996 ; 12 : 1129-40.
22. Holl V, Hemmerter S, Burrer R, et al. Involvement of Fc gamma RI (CD64) in the mechanism of HIV-1 inhibition by polyclonal $\lg G$ purified from infected patients in cultured monocyte-derived macrophages. J Immunol 2004 ; 173 : 6274-83.

23. Lambotte 0. Les patients HIV controllers. Med Sci (Paris) $2012 ; 28: 172-8$.

24. Mestecky J, Jackson S, Moldoveanu Z, et al. Paucity of antigen-specific IgA responses in sera and external secretions of HIV-type l-infected individuals. AIDS Res Hum Retrovir $2004 ; 20$ : 972-88.

25. Broliden K, Hinkula J, Devito C, et al. Functional HIV-1 specific IgA antibodies in HIV-l exposed, persistently IgG seronegative female sex workers. Immunol Lett $2001 ; 79: 29-36$.

26. Mantis NJ, Palaia J, Hessell AJ, et al. Inhibition of HIV-1 infectivity and epithelial cell transfer by human monoclonal $\lg G$ and IgA antibodies carrying the b12 V region. J Immunol 2007 ; 179 : 3144-52.

27. Stieh G, McRaven M, Cianci G, et al. Development of an imaging based virus aggregation assay for vaccine development. Retrovirology $2012 ; 9: 319$.

28. Watkins JD, Sholukh AM, Mukhtar MM, et al. Anti-HIV IgA isotypes: differential virion capture and inhibition of transcytosis are linked to prevention of mucosal R5 SHIV transmission. AIDS $2013 ; 27$ : F13-F20.

29. Laurence J, Saunders A, Early $\varepsilon$, Salmon JE. Human immunodeficiency virus infection of monocytes: relationship to $\mathrm{Fc}$-gamma receptors and antibodydependent viral enhancement. Immunology $1990 ; 70: 338-43$.

30. Takada A, Kawaoka Y. Antibody-dependent enhancement of viral infection: molecular mechanisms and in vivo implications. Rev Med Virol 2003; 13 : 387-98.

31. Forthal DN, Gabriel દદ, Wang A, et al. Association of Fcgamma receptor Illa genotype with the rate of HIV infection after gp120 vaccination. Blood $2012 ; 120: 2836-42$.

32. Haynes BF, Kelsoe G, Harrison SC, Kepler TB. B-cell-lineage immunogen design in vaccine development with HIV-1 as a case study. Nat Biotechnol $2012 ; 30: 423-33$.

33. Moldt B, Schultz N, Dunlop DC, et al. A panel of $\operatorname{lgGl}$ bl2 variants with selectively diminished or enhanced affinity for Fcgamma receptors to define the role of effector functions in protection against HIV. J Virol $2011 ; 85$ : 10572-81.

34. Moldt B, Shibata-Koyama M, Rakasz $\varepsilon G$, et al. A nonfucosylated variant of the anti-HIV-1 monoclonal antibody b12 has enhanced FcgammaRIIlamediated antiviral activity in vitro but does not improve protection against mucosal SHIV challenge in macaques.J Virol $2012 ; 86: 6189-96$.

35. Ruffin N, Borggren M, Euler Z, et al. Rational design of HIV vaccines and microbicides: report of the EUROPRISE annual conference 2011. J Transl Med $2012 ; 10: 144$.

36. PX'Therapeutics. PX'Therapeutics annonce l'essai clinique EuroNeut-41 portant sur un vaccin prophylactique contre le VIH. Business Wire 2012 ; 12 mars.

37. $\mathrm{Ng} C \mathrm{CT}$, Jaworski JP, Jayaraman $\mathrm{P}$, et al. Passive neutralizing antibody controls SHIV viremia and enhances $B$ cell responses in infant macaques. Nat Med $2010 ; 16: 1117-9$.

38. Michaud HA, Gomard T, Gros L, et al. A crucial role for infected-cell/ antibody immune complexes in the enhancement of endogenous antiviral immunity by short passive immunotherapy. PLoS Pathog $2010 ; 6$ : el 000948.

39. Abes R, Gelize $\varepsilon$, Fridman WH, Teillaud JL. Long-lasting antitumor protection by anti-CD20 antibody through cellular immune response. Blood $2010 ; 116$ : 926-34.

40. Schwartz C, Le Douce V, Cherrier T, et al. Un virus tapi dans l'ombre : les bases moléculaires de la latence du VIH-1. Med Sci (Paris) $2010 ; 26$ : 159-64.

41. Le Tortorec A, Dejucq-Rainsford N. Le tractus génital masculin. Med Sci (Paris) $2010 ; 26: 861-8$.

42. Abès R, Dutertre CA, Teillaud JL. Les anticorps : mieux les connaître pour mieux s'en servir. Med Sci (Paris) 2009 ; 25 : 1011-9.

43. Deligne C, Teillaud JL. Le double visage des anticorps monoclonaux en oncologie. Med Sci (Paris) 2013 ; $29: 57-63$.
TIRÉS À PART

C. Moog 